\title{
Isolation Enhancement of Compact Dual-Band Printed MIMO Antenna for WLAN and 5G Applications
}

\author{
Adham M. Salah ${ }^{1}$, Majid S. A. Alkhambashi ${ }^{2}$, Naem A. Jan ${ }^{1}$, Tariq A. Nagem ${ }^{1}$, Raed A. Abd-Alhameed ${ }^{1,3}$ \\ \{amssaleh@bradford.ac.uk ${ }^{1}$, majid@majidtek.com ${ }^{2}$, kakarnayeem@yahoo.com ${ }^{3}$ \} \\ ${ }^{1,3}$ School of Engineering and Informatics, University of Bradford, Richmond Rd, Bradford BD7 1DP \\ ${ }^{2}$ Sultanate of Oman- Muscat B.Box:380 PC: 318 \\ ${ }^{3}$ Information and Communication Eng. Department, Basrah University College of Science and \\ Technology, Basrah 24001, Iraq
}

\begin{abstract}
A compact dual-band $(2 \times 2)$ MIMO antenna that operates at $2.4 \mathrm{GHz}, 3.4 \mathrm{GHz}$ and $3.6 \mathrm{GHz}$ for wireless local area network (WLAN) and Sub-6GHz $5^{\text {th }}$ generation $(5 \mathrm{G})$ applications is demonstrated in this paper. A metamaterial resonator method for improving the isolation is applied by introducing four SRRs resonating at $2.4 \mathrm{GHz}$ between the adjusting elements. The overall size of the antenna is $46 \times 54 \times 1.6 \mathrm{~mm}^{3}$. The proposed antenna achieves impedance bandwidths from $2.15 \mathrm{GHZ}$ to $2.75 \mathrm{GHz}$ and from $3.1 \mathrm{GHz}$ to $4.4 \mathrm{GHz}$ for the two bands respectively. The reported isolation is $30 \mathrm{~dB}$ around the $2.5 \mathrm{GHz}$ band and $20 \mathrm{~dB}$ around the $3.5 \mathrm{GHz}$ band. The features of the proposed MIMO antenna are illustrated in terms of s-parameters, ECC, TARC, CCL, DG and antenna radiation patterns. These parameters are analyzed carefully and the consequences show that the proposed MIMO antenna is suitable to work in the intended frequency bands.
\end{abstract}

Keyword: MIMO Antenna, Metamaterial Resonator, SRR, Mutual Coupling, $5^{\text {th }}$ generation

\section{Introduction}

Recently, the communications systems are expanded explosively to support the increasing demands of high transmission rate with high throughput for various fixed and mobile services. To fulfill these demands, the researchers and the manufacturers have been shifted to the fifth-generation (5G) technology. Multiple Input Multiple Output (MIMO) system can be considered as the backbone of the 5G technology due to its unique features of achieving higher data rates without consuming extra power in the multipath environment $[1,2]$. One significant drawback associated with the MIMO system is the mutual coupling between the radiating elements inside the MIMO antenna structure. This means that some of the energy will be radiated from one element to the other one and vice versa. The main reason for this issue is the limited area between the multiple radiating antennas inside the MIMO structure. Therefore, if the radiating elements are farther the isolation between them will be stronger $[3,4]$.

Various techniques have been developed in the last few years to enhance the isolation inside the MIMO antenna structure. Various antenna designs with different operating frequency bands such as narrowband, wideband and dual/triple band [5] have used these approaches. Methods reported include using parasitic elements between the antennas [6,7], junctions and branches in the form of T-shape [8,9], defected ground structures (DGS) [10,11], stubs [11, 12], neutralization lines (NL) [13-17] and metamaterial resonators [1825].

This last method uses metamaterial features of controlling electromagnetic wave propagation to increase the isolation and improve the MIMO antenna performance. This could be achieved by inserting metamaterial resonators between the radiating antennas of the MIMO antenna structure [26, 27].

In this presented work, a two-band 2x2 MIMO antenna with two arc-shaped radiating elements has been modeled and simulated to fulfill the requirements of WLAN and Sub-6GHz 5G applications. The proposed structure is excited using the feeding technique of coplanar waveguide (CPW) and is fabricated on FR4 substrate. Four metamaterial resonators in the form of the split-ring resonator (SRR) are applied to enhance the isolation inside the MIMO antenna structure. The MIMO antenna performance characteristics are studied using simulation and measurement in terms of scattering parameters, ECC, TARC, CCL, DG and antenna radiation pattern. The analysis of these outcomes explains that the proposed design is convenient for the two intended frequency bands. 


\section{The Specifications of the SRR}

Generally, the behavior of the spite ring resonator (SRR) could be treated as an electric dipole that can be excited by external electric flux. In the MIMO antenna application, the presence of SRR with its feature of open rings between the radiating elements will lead to produce an effective permeability with negative value at the resonant frequency which in turn can control its electric and magnetic features. Practically, it has been explained that an SRR can be inserted between two individual antennas in MIMO system to enhance the isolation [28].

The configuration of the unit cell in the form of SRR is illustrated in Fig. (1). The SRR is modelled and simulated using CST Microwave Studio depending on [29] while the Matlab program is used here to calculate constitutive values of the SRR, for instance, effective permittivity and permeability. It could be easily seen from Fig. (1) that the unit cell in this paper has symmetrical periodicity in the armature. This leads to generate negative values of permittivity and permeability at the same time as explained in Fig. (2). On the other hand, an SRR with no periodicity usually produces negative permeability only as shown in [25].

Basically, the main usage of the metamaterial resonator in this paper is to minimize the coupling between the radiating antennas inside the MIMO antenna structure. Therefore, four SRRs of size $9 \mathrm{~mm} \times 5.5 \mathrm{~mm}$ are inserted between the radiating elements of the proposed MIMO antenna. The optimized dimensions of the proposed SRR are listed in Table 1. The s-parameters in terms of S11 and S21 of the SRR are explained in Fig. (3), it could be clearly seen that the SRR is resonating at $2.45 \mathrm{GHz}$.

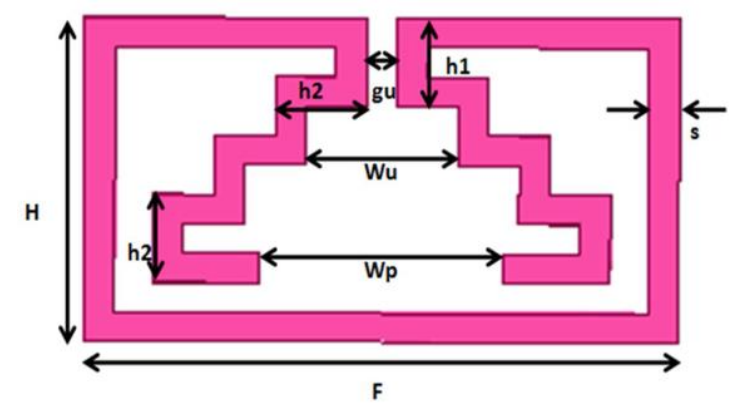

Figure 1. The geometrical configuration of the SRR.

Table 1. The SRR unit cell optimized dimensions.

$\begin{array}{cccc}\text { Parameters } & \text { Value } & \text { Parameter } & \text { value } \\ \text { H } & 5.5 \mathrm{~mm} & \mathrm{Wp} & 4 \mathrm{~mm} \\ \text { F } & 9.75 \mathrm{~mm} & \mathrm{Wu} & 2.5 \mathrm{~mm} \\ \text { h1 } & 1.5 \mathrm{~mm} & \mathrm{gu} & 0.5 \mathrm{~mm} \\ \text { h2 } & 1.5 \mathrm{~mm} & \mathrm{~s} & 0.5 \mathrm{~mm}\end{array}$

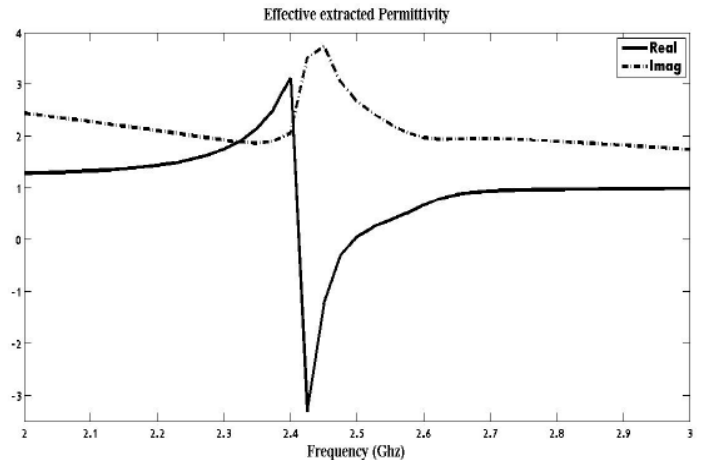

(a)

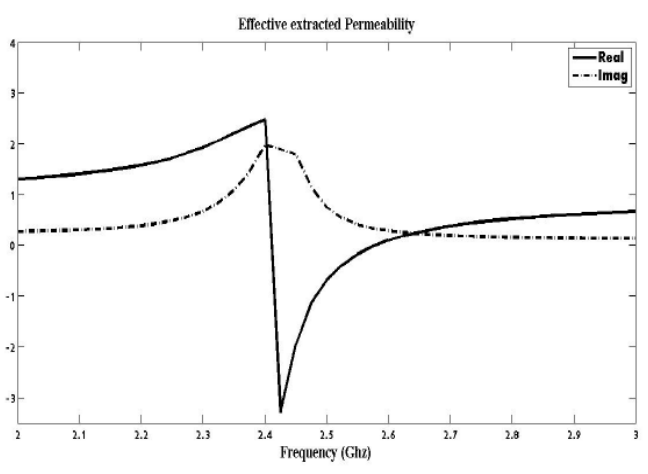

(b) 
Figure 2. The effective values of the proposed SRR; (a) effective permittivity, (b) effective permeability.

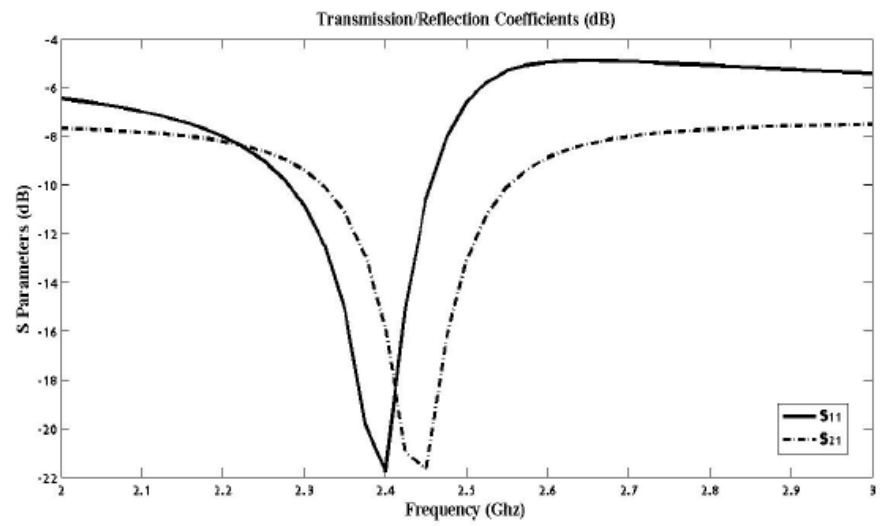

Figure 3. Transmission/Reflection coefficient of the proposed SRR unit cell.

\section{The antenna layout and procedure}

A two-band 2x2 MIMO antenna system with two arc-shaped radiating antennas is modeled and simulated to fulfill the requirements of WLAN and Sub-6GHz $5 \mathrm{G}$ applications. The simple approach to design an antenna with a dual-band operation is by obtaining two elements with different lengths inside the antenna structure. So that, each element will resonate at the desired frequency by matching its length with a quarter of the wavelength of the operating frequency [30]. Depending on the procedure described in [31], the total length of the long arcshaped radiating element is set to be $23.25 \mathrm{~mm}$ to work at $2.45 \mathrm{GHz}$ while the total length of the short arc-shaped radiating element is set to be $12 \mathrm{~mm}$ to work at $3.4 \mathrm{GHz}$. The substrate parameters are characterized by a thickness of $1.6 \mathrm{~mm}$, a relative permittivity of 4.3 , and a tangent loss of 0.025 . The overall dimensions of the MIMO antenna are $54 \times 46 \times 1.6 \mathrm{~mm}^{3}$. CPW lines are used to feed the radiating antennas of the MIMO antenna system. The space between the nearest edges of the two radiating antennas is equal to $6.65 \mathrm{~mm}\left(0.0532 \lambda_{0}\right.$ at $2.4 \mathrm{GHz}$ ). Four SRR metamaterial unit cells are introduced between the two radiating antennas to minimize the unwanted coupling as explained in Fig. (4). The optimum dimensions of Fig. (4) are listed in Table 2.

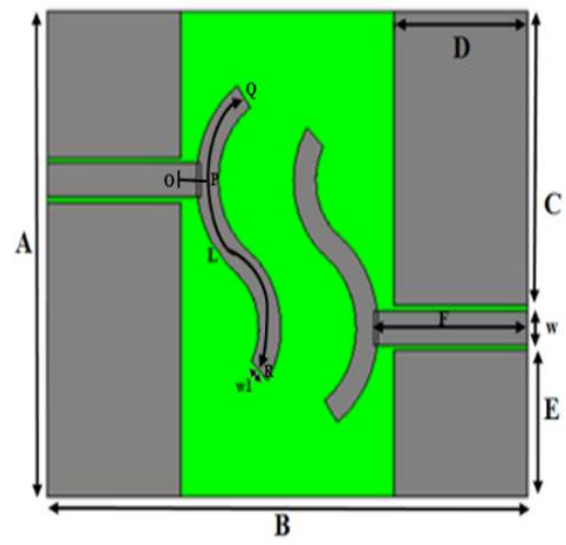

(a)

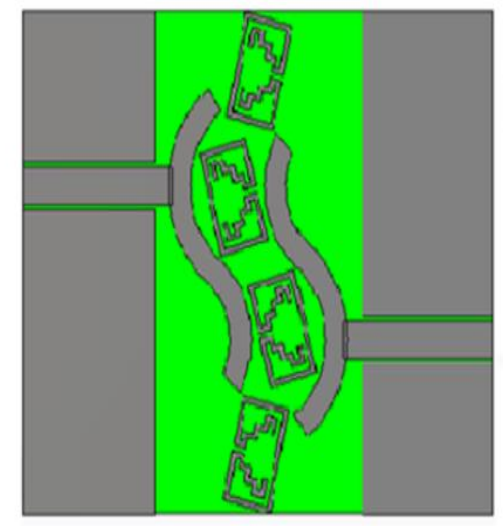

(b)

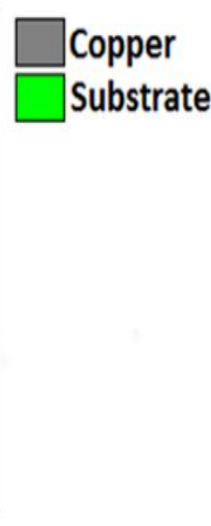

Figure 4. Configurations of the presented MIMO antenna; (a) antenna without metamaterial resonators (b) antenna with metamaterial resonators. 
Table 2. The presented MIMO

$\begin{array}{cccc}\text { Parameters } & \text { Value } & \text { Parameter } & \text { value } \\ \text { A } & 46 \mathrm{~mm} & \text { F } & 17.2 \mathrm{~mm} \\ \text { B } & 54 \mathrm{~mm} & \text { W } & 3.2 \mathrm{~mm} \\ \text { C } & 27.9 \mathrm{~mm} & \text { w1 } & 2.5 \mathrm{~mm} \\ \text { D } & 15 \mathrm{~mm} & \text { OPQ } & 12 \mathrm{~mm} \\ \text { E } & 13.9 \mathrm{~mm} & \text { OPR } & 23.25 \mathrm{~mm}\end{array}$

antenna optimized dimensions.

\section{Parametric study}

In order to explain the impact of inserting the SRRs between the radiating elements and achieve the optimum results of the presented MIMO design, a parametric study was carried out in the following section depending on the number of SRR unit cells between the radiating antennas of the presented MIMO antenna.

\subsection{Scattering-Parameters of the presented Design with and without SRRs}

The effectiveness of the metamaterial resonators has been validated by comparing the simulated sparameters of the proposed MIMO antenna in two different cases (with and without metamaterial resonators). Figure (5) explains the simulated outcomes of the reflection and transmission coefficients. It is clearly obvious in Fig. (5-a) that the two frequency bands still appeared after inserting the unit cells and there is bandwidth extension in the two operating frequencies. Moreover, the mutual coupling has decreased in the first band with the existence of the metamaterial resonators and it reaches to $-35 \mathrm{~dB}$ around $2.5 \mathrm{GHz}$ while the dip of the second band has shifted toward the first band but it still achieves isolation around $15 \mathrm{~dB}$ at $3.4 \mathrm{GHz}$ and $3.6 \mathrm{GHz}$ as illustrated in Fig. (5.b).
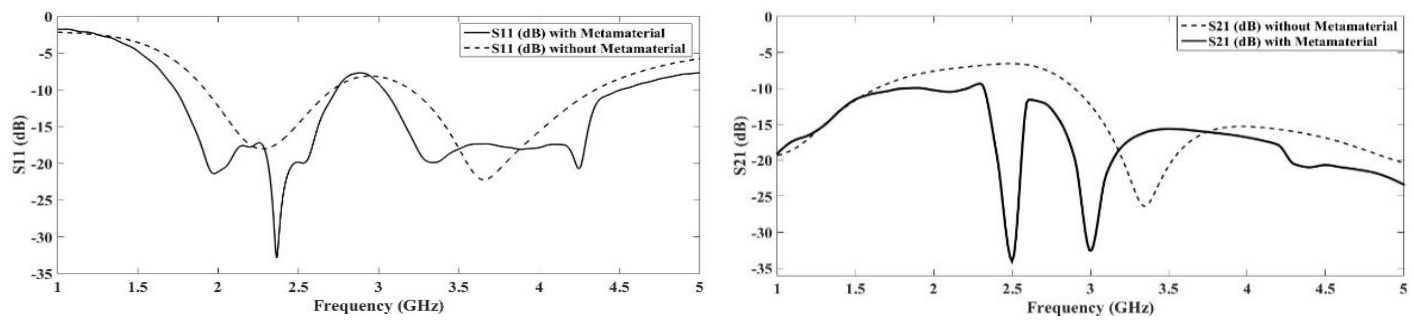

Figure 5. Simulated s-parameters of the presented MIMO antenna with a different number of metamaterials resonators. (a) reflection coefficients and (b) transmission coefficients.

\subsection{Scattering-Parameters of the presented Design with three variations of metamaterial resonators}

In this section, three different cases are studied to validate the effectiveness of the number of metamaterial resonators inside the antenna structure. The simulated s-parameters of the antenna with different numbers of metamaterial resonators (SRRs) are illustrated in Fig. (6). Figure (6-b) shows that increasing the number of metamaterial resonators will lead to decrease the mutual coupling around $2.4 \mathrm{GHz}$ and to reach $-35 \mathrm{~dB}$ with four metamaterial resonators. While the two bands still appear with S11 less than $-10 \mathrm{~dB}$ as shown in Fig. (6-a).

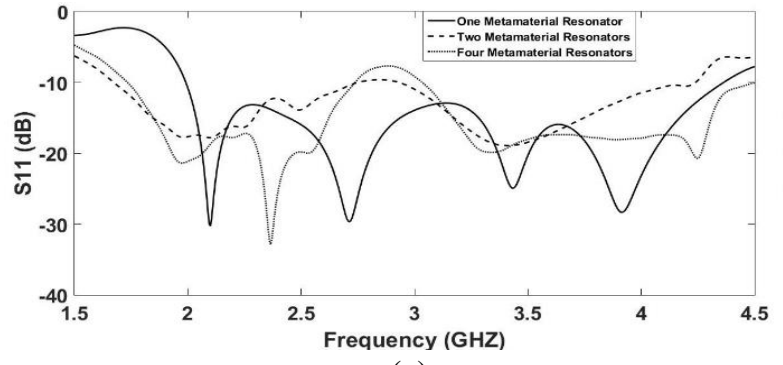

(a)

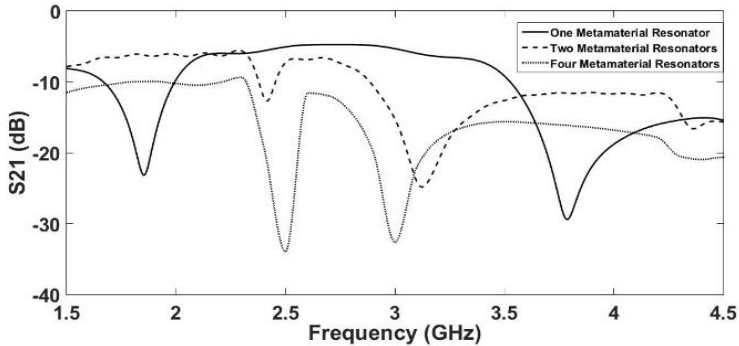

(b) 
Figure 6. Simulated s-parameters of the presented MIMO antenna with a different number of metamaterials resonators; (a) reflection coefficients and (b) transmission coefficients.

\section{Practical and Calculated Results Discussions}

To verify the outcomes of the simulation stage, the proposed design of the MIMO antenna was fabricated and tested. The prototype of this design is displayed in Fig. (7). Vector network analyzer (VNA) is used to test the scattering parameters in terms of S11and S21 while the radiation patterns are tested using the anechoic chamber. These measurement results have been used to analyze the performance of the proposed MIMO antenna. The measured achievements in terms of scattering parameters, envelope correlation coefficient (ECC), (TARC), capacity loss (CL), radiation pattern and diversity gain (DG) are explained in the following paragraphs.

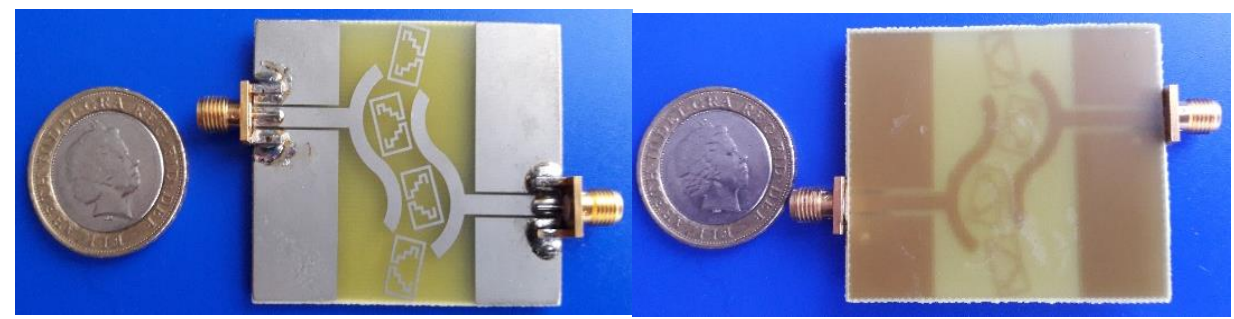

(a)

(b)

Figure 7. The photograph of the fabricated MIMO design, (a) The front view of the MIMO; (b)The back view of the MIMO.

The comparison between the calculated and the measured results of S11 and S21 is illustrated in Fig. (8). This figure demonstrates the matching degree between the two results. A fair agreement between two results is achieved with some variance due to the measurement tolerances. The practical values of S11 versus frequency are shown in Fig. (8-a). It can be clearly seen from this figure that a $-10 \mathrm{~dB}$ impedance bandwidths are obtained from $2.15 \mathrm{GHz}$ to $2.75 \mathrm{GHz}$ at the lower resonant frequency band and from $3.1 \mathrm{GHz}$ to $4.4 \mathrm{GHz}$ at the upper resonant frequency band. The mutual coupling values are $-30 \mathrm{~dB}$ at $2.4 \mathrm{GHz}$ and $-20 \mathrm{~dB}$ at $3.4 \mathrm{GHz}$ and $-19 \mathrm{~dB}$ at $3.6 \mathrm{GHz}$ frequency.
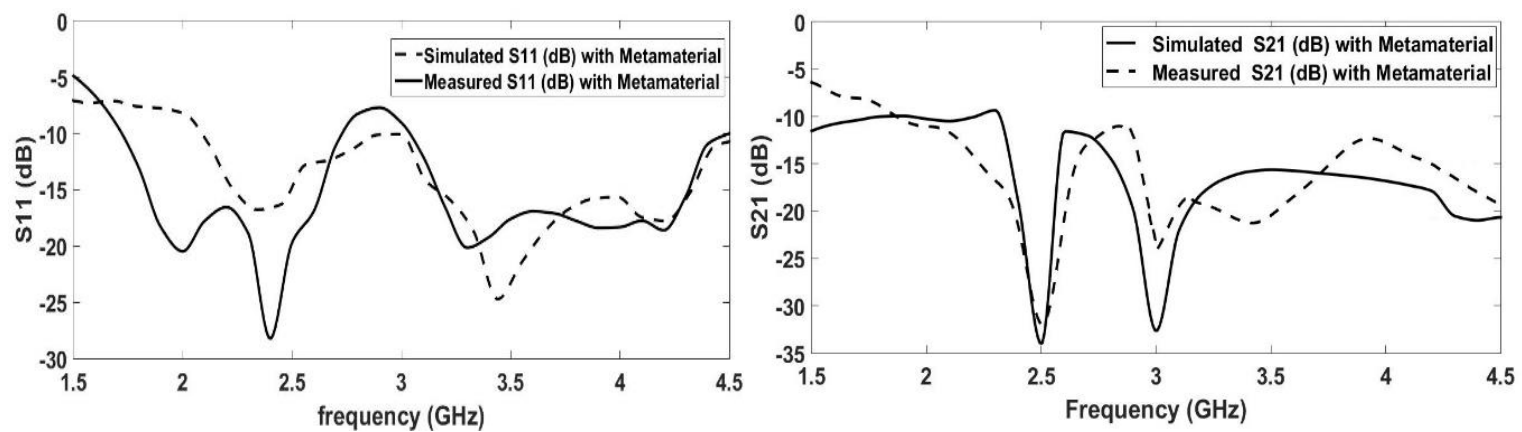

Figure 8. Simulated and measured results of the proposed MIMO antenna (a) Reflection coefficient, S11 (b) Transmission coefficient, S21.

Generally, the envelope correlation coefficient (ECC) can play a significant factor in MIMO antenna systems. This value could be evaluated through two different approaches. The first approach depends on the far-field radiation pattern of the antenna $[1,32]$ and the second approach uses the s-parameters of the MIMO antenna [33]. The simulated and measured ECC of the proposed MIMO antenna are calculated depending on the second approach using equation 1 [33] and the consequences are illustrated in Fig. (9). It can be clearly noticed from Fig. (9) that the value of the ECC is less than 0.1 in two bands which is significantly less than the 0.3 , the requirement for MIMO antenna applications [34]. 


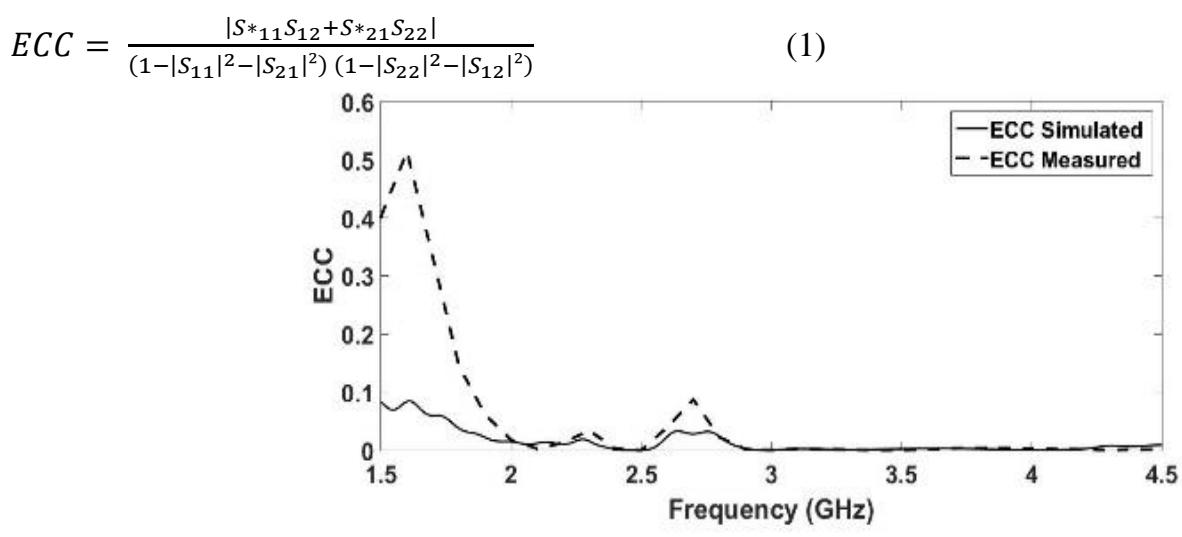

Figure 9. Simulated and measured the envelope correlation coefficient.

Basically, the capacity of the channel of the MIMO system could be enhanced by increasing the number of radiating antennas. On the other hand, the existence of the uncorrelated Rayleigh-fading may lead to a loss in the channel capacity.

For two elements of MIMO systems, the channel capacity loss could be calculated by using the correlation matrix mentioned in [35]. The simplified form of channel capacity loss is listed below [36].

$$
C_{\text {loss }}=-\log _{2} \operatorname{det}\left(\varphi^{R}\right)
$$

where $\varphi^{R}$ is the correlation matrix receiving antenna:

$$
\begin{gathered}
\varphi^{R}=\left[\begin{array}{ll}
\rho_{11} & \rho_{12} \\
\rho_{21} & \rho_{22}
\end{array}\right] \\
\text { with } \rho_{11}=\left(1-\left(\left|S_{11}\right|^{2}+\left|S_{12}\right|^{2}\right)\right) \\
\text { and } \rho_{12}=-\left(S_{11}^{*} S_{12}+S_{21}^{*} S_{22}\right)
\end{gathered}
$$

The computed and measured capacity loss of the presented MIMO antenna is illustrated in Fig. (10). In this figure, the capacity loss is less than $0.5 \mathrm{bit} / \mathrm{s} / \mathrm{Hz}$ for the two bands and there is a very good agreement between the calculated and measured outcomes.

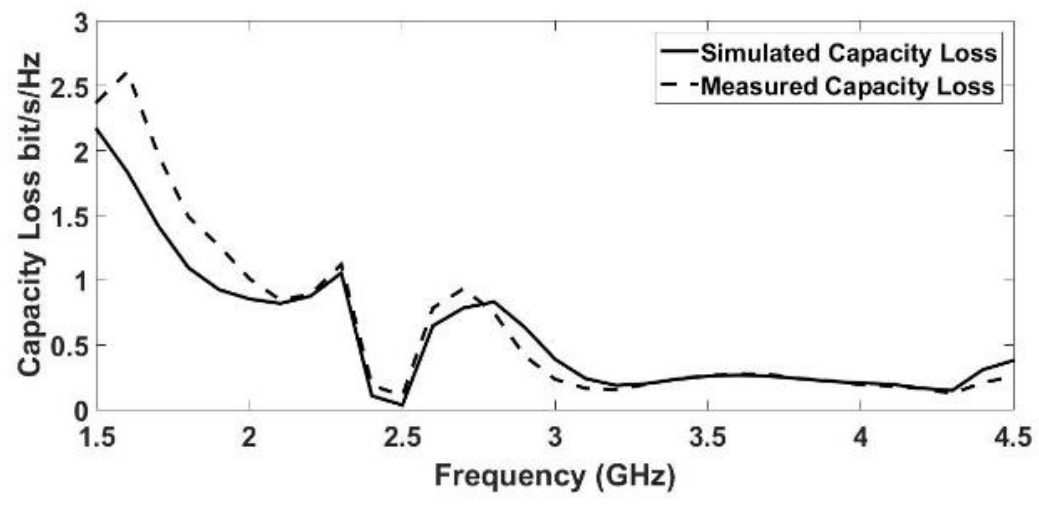

Figure 10. Simulated and measured capacity loss of the proposed MIMO antenna.

The total active reflection coefficient (TARC) is another significant parameter in the MIMO antenna system which is evaluated to properly describe the bandwidth and the efficiency of the system. TARC defines as the result of dividing the square root of the total reflected power to the square root of the total incident power [34]. Equation 3 uses to evaluate the TARC value in the two-port MIMO antenna system [14].

$$
\tau_{a}^{t}=\sqrt{\frac{\left(\left(\left|S_{11}+S_{12} e^{j \theta}\right|^{2}\right)+\left(\left|S_{21}+S_{22} e^{j \theta}\right|^{2}\right)\right)}{2}}
$$


The computed TARC value of the suggested MIMO antenna is explained in Fig. (11). Figure (11-a) has been created using equation 3 to cover the phase range from 0 to 180 degrees with phase steps of 30 degrees. The average value of TARC is evaluated using Matlab package and it is presented in Fig (11-b). The computed TARC values shown in Fig. (11) indicate that the two required bands are covered with a TARC value less than $-10 \mathrm{~dB}$.
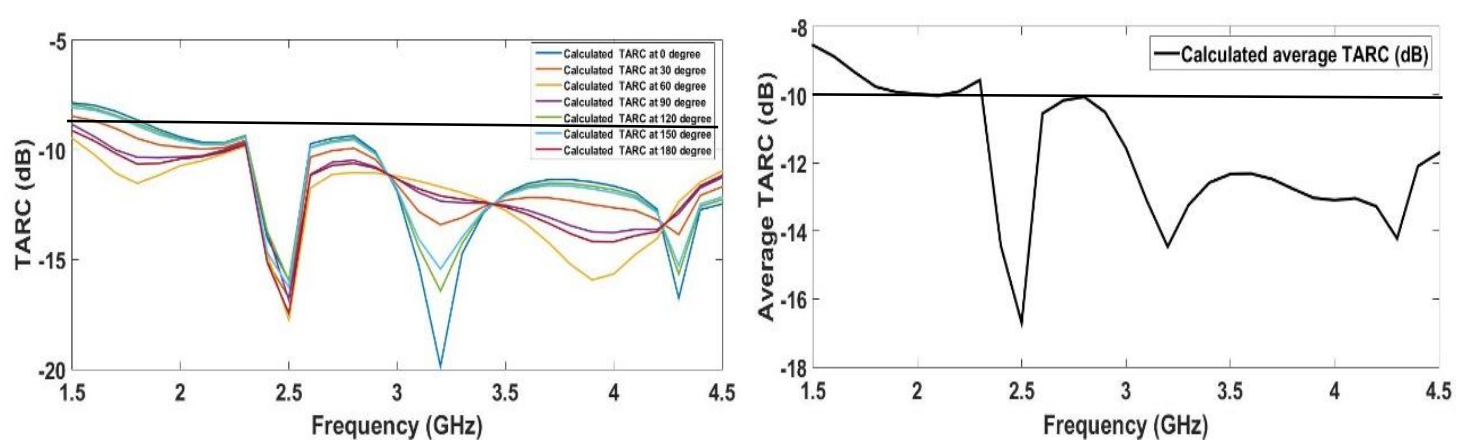

Figure 11. Calculated values of TARC (a) TARC with different phase; (b) Average value of TARC.

The prototyped module of the presented MIMO antenna is tested to evaluate the radiation patterns in the far-field region at the two required frequency bands (WLAN and 5G). These patterns were measured in the two planes of XZ and YZ, in the case of exciting port 1 and terminating port 2 . The practical results are shown in Fig. (8) and they explain that at the two frequency bands the far-field radiations achieve stable omnidirectional patterns.

Diversity Gain (DG) is a major parameter that influences the system performance of a MIMO antenna. This metric can be understood as the amount of improvement in the diversity antenna system in one diversity channel compared to an antenna system of a single element [34]. In this work, the values of the simulated DG are presented in Fig. (13) and it is clearly seen that the DG values for the two frequency bands are very close to the ideal value of $\mathrm{DG}(\mathrm{DG}=10 \mathrm{~dB})$.

Furthermore, the proposed MIMO antenna has been compared with several published data that used SRR as a coupling reduction technique. This comparison is not comprehensive but it has a fair representative for the recent state of the art of this technology. The comparison depends on the overall size of the antenna, the band of the operating frequency, the space between the radiating antennas and finally the isolation between the radiating antennas. The summary of this comparison is listed in Table 3. This comparison shows that the isolation of the proposed MIMO antenna is larger than other designs compared to the separation distance of the antennas. 

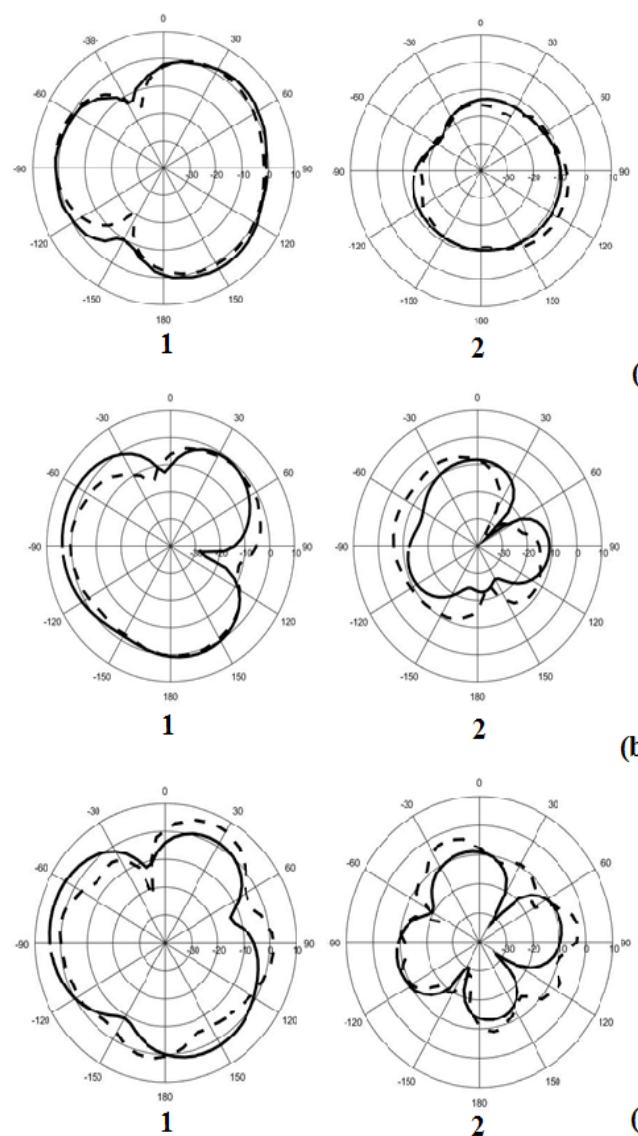

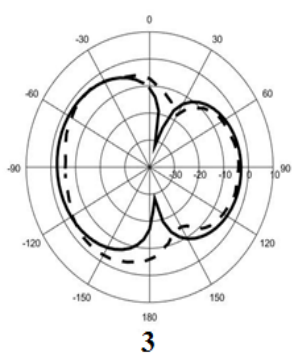

(a)

(b)
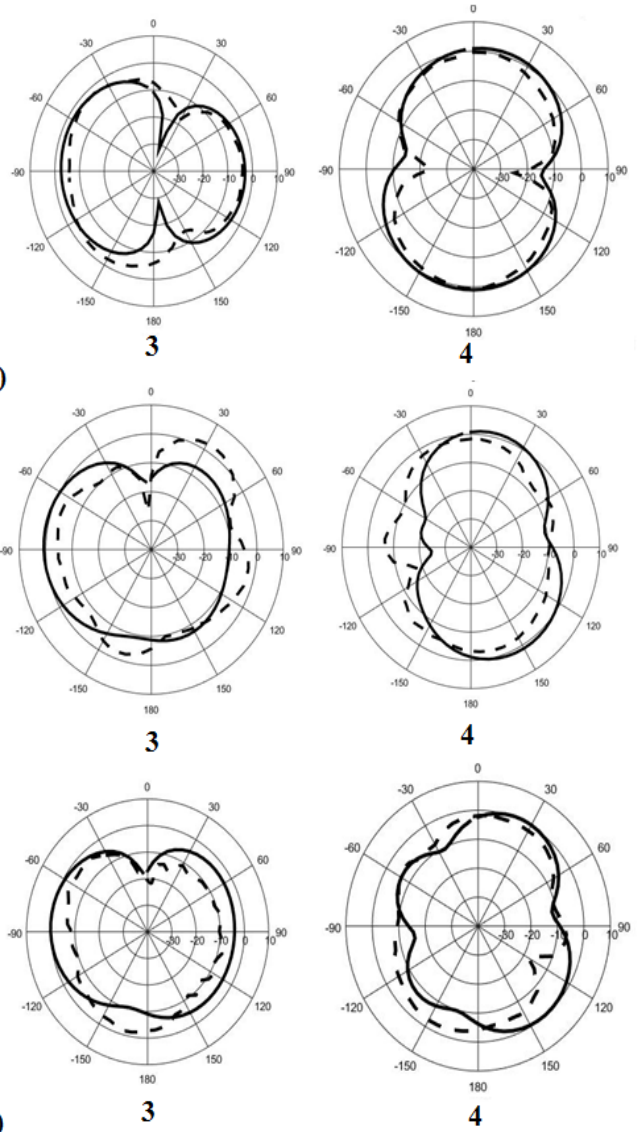

(c)

Figure 12. calculated and measured $2 \mathrm{D}$ electromagnetic radiation patterns of the presented MIMO antenna design in two different planes [(1 and 2) y-x plane and (3 and 4) z-x plane] at (a) $2.4 \mathrm{GHz}$ (b) $3.4 \mathrm{GHz}$ and (c) $3.6 \mathrm{GHz}$. Port 1 is excited and port 2 is terminated. " calculated results, " 4" cross-polar components.

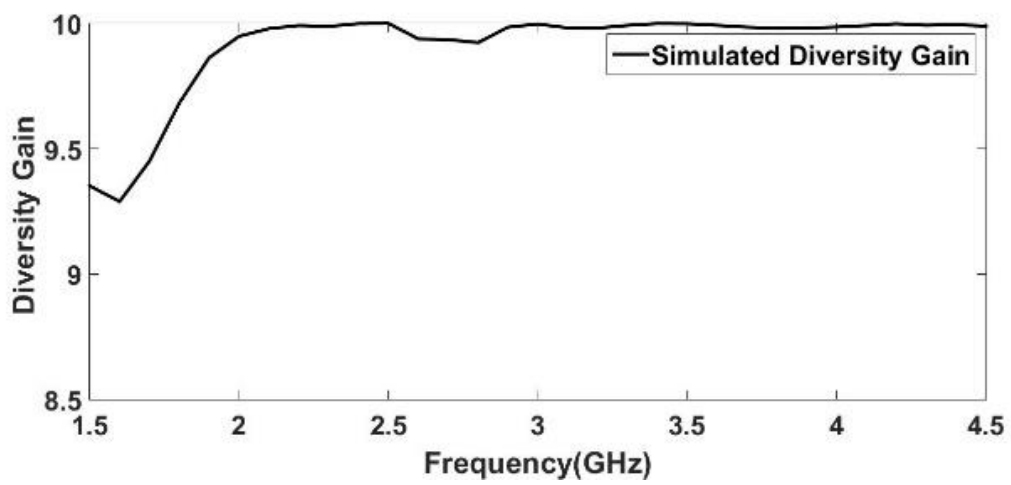

Figure 13. The variation of diversity gain versus frequency of the proposed MIMO antenna.

Table 3. Comparison between this work and other researchers works.

$\begin{array}{ccccc}\text { Reference } & \text { Size }\left(\mathbf{m m}^{3}\right) & \begin{array}{c}\text { Frequency range } \\ (\mathbf{G H z})\end{array} & \begin{array}{c}\text { Separation } \\ \text { distance }\end{array} & \begin{array}{c}\text { Isolation } \\ (\mathbf{d B})\end{array} \\ \mathbf{1 8} & 78 \times 60 \times 1.27 & 4.85-5.1 & 0.25 \lambda_{\mathrm{o}} & 26 \\ \mathbf{1 9} & 84 \times 44 \times 1 & 3.65-3.75 & 0.5 \lambda_{\mathrm{o}} & 42 \\ \mathbf{2 1} & 100500.8 & 2.45-2.5 & 0.17 \lambda_{\mathrm{o}} & 10 \\ \mathbf{2 2} & 60 \times 60 \times 1.6 & 2.4-2.475 & \mathrm{NA} & 22 \\ \text { This work } & 54 \times 46 \times 1.6 & \begin{array}{c}2.15-2.75 ; 3.1- \\ 4.4\end{array} & 0.0532 \lambda_{\mathrm{o}} & 30,20 \\ & & & & \end{array}$




\section{Conclusion}

In this presented work, a two-band 2x2 MIMO antenna for wireless LAN and Sub-6GHz 5G applications has been designed. Metamaterial resonators in the form of SRRs unit cells have been added between the radiating antennas of the MIMO design for decoupling purposes. The coupling has been optimized by changing the number of SRRs between the radiating antennas of the presented MIMO antenna design. It has been obvious that increasing the number of the SRR inside the MIMO antenna design and in accurate positions will lead to improve isolation and in turn improve the system performance. The proposed MIMO antenna is fabricated and tested in terms of the scattering parameters, ECC, TARC, capacity loss, DG and antenna radiation patterns. These parameters are analyzed carefully and the consequences show that the presented MIMO design is suitable to work in the intended frequency bands.

\section{Acknowledgements}

Adham M. Saleh would like to thank the Ninevah University and the Higher Committee for Education Development in Iraq (HCED) for supporting this project financially and monitoring his Ph.D. progress. The authors would like to thank Ninevah University and the University of Bradford for their massive assist during this project.

\section{References}

[1] R. G. Vaughan and J. B. Andersen, "Antenna diversity in mobile communications," IEEE Transactions on Vehicular Technology, vol. VT-36, pp. 147-172, Nov. 1987.

[2] G. J. Foschini and M. J. Gans, "On limits of wireless communications in a fading environment when using multiple antennas, " Wireless Pers. Commun., vol. 6, pp. 311-335, 1998.

[3] J. Leivo," Improving the performance of strongly coupled antennas using a compensating transmission line network", MSc Thesis, Gothenburg, Chalmers University of Technology, Sweden,2009.

[4] S. R.Saunders, A. A. Zavala, "Antennas and propagation for wireless communication systems", JohnWiley \& Sons Ltd, Second Edition, 2007.

[5] C.H.See, et al. (2018) "Compact Wideband Printed MIMO/Diversity Monopole Antenna for GSM/UMTS and LTE Applications", In: Elfergani I., Hussaini A., Rodriguez J., Abd-Alhameed R. (eds) Antenna Fundamentals for Legacy Mobile Applications and Beyond. Springer, Cham.

[6] R. Addaci, A. Diallo, C. Luxey, P. Le Thuc and R. Staraj, "Dual-Band WLAN Diversity Antenna System With High Port-to-Port Isolation," in IEEE Antennas and Wireless Propagation Letters, vol. 11, pp. 244-247, 2012.

[7] X. b. Sun and M. Y. Cao, "Low mutual coupling antenna array for WLAN application," in Electronics Letters, vol. 53, no. 6, pp. 368-370, 2017.

[8] X. L. Liu, Z. Wang, Y.-Z. Yin, and J. H. Wang, "Closely spaced dual band-notched UWB antenna for MIMO applications," Progress In Electromagnetics Research C, vol. 46, 2014, PP. 109-116.

[9] H. Qin and Y.-F. Liu, "Compact dual-band MIMO antenna with high port isolation for WLAN applications", Progress In Electromagnetics Research C, Vol. 49, 2014, PP.97-104.

[10] P. Sharmaa and T. Khanb, "A Compact MIMO Antenna with DGS Structure," International Journal of Current Engineering and Technology, Vol.3, No.3, pp. 780-782, 2013.

[11] W. N. N.W. Marzudi, et al., "Uni-Planer MIMO Antenna for WLAN and WiMAX Wireless Services," International Journal of Computer and Information Technology, vol. 8, Issue 3, pp. 78-83, May 2019

[12] H. Zhao, F. Zhang, C. Wang, and J. Liang, "A Compact UWB Diversity Antenna," International Journal of Antennas and Propagation, vol. 2014, Article ID 805853, 6 pages, 2014.

[13] W.N.N.W. Marzudi, et al.,(2015) "Two-Elements Crescent Shaped Printed Antenna for Wireless Applications", In: Sulaiman H., Othman M., Othman M., Rahim Y., Pee N. (eds) Advanced Computer and Communication Engineering Technology. Lecture Notes in Electrical Engineering, vol 315. Springer, Cham

[14] S. Su, C. Lee and F. Chang, "Printed MIMO-Antenna System Using Neutralization-Line Technique for Wireless USB-Dongle Applications", in IEEE Transactions on Antennas and Propagation, vol. 60, no. 2, pp. 456-463, Feb. 2012.

[15] Y. Wang and Z. Du, "A Wideband Printed Dual-Antenna With Three Neutralization Lines for Mobile Terminals", in IEEE Transactions on Antennas and Propagation, vol. 62, no. 3, pp. 1495-1500, March 2014.

[16] S. Zhang and G. F. Pedersen, "Mutual Coupling Reduction for UWB MIMO Antennas With a Wideband Neutralization Line", in IEEE Antennas and Wireless Propagation Letters, vol. 15, no., pp. 166-169, 2016.

[17] A. M. Saleh, K. H. Sayidmarie, R. A. Abd-Alhameed, S. Jones, J. M. Noras and P. S. Excell, "Compact tri-band MIMO antenna with high port isolation for WLAN and WiMAX applications", 2016 Loughborough Antennas \& Propagation Conference (LAPC), Loughborough, 2016, pp. 1-4. 
[18] R. Selvaraju, M. H. Jamaluddin, M. R. Kamarudin, J. Nasir, and M. H. Dahri, "Complementary split ring resonator for isolation enhancement in 5G communication antenna array", Prog. Electromagn. Res., vol. 83, pp. 217-228, 2018.

[19] M. M. Bait-Suwailam, O. F. Siddiqui and O. M. Ramahi, "Mutual Coupling Reduction Between Microstrip Patch Antennas Using Slotted-Complementary Split-Ring Resonators", in IEEE Antennas and Wireless Propagation Letters, vol. 9, pp. 876-878, 2010

[20] Z. Qamar, L. Riaz, M. Chongcheawchamnan, S. A. Khan and M. F. Shafique, "Slot combined complementary split ring resonators for mutual coupling suppression in microstrip phased arrays", in IET Microwaves, Antennas \& Propagation, vol. 8, no. 15, pp. 1261-1267, 9122014.

[21] M. S. Sharawi, M. U. Khan, A. B. Numan and D. N. Aloi, "A CSRR Loaded MIMO Antenna System for ISM Band Operation", in IEEE Transactions on Antennas and Propagation, vol. 61, no. 8, pp. 4265-4274, Aug. 2013.

[22] A. Ramachandran, S. Valiyaveettil Pushpakaran, M. Pezholil and V. Kesavath, "A Four-Port MIMO Antenna Using Concentric Square-Ring Patches Loaded With CSRR for High Isolation", in IEEE Antennas and Wireless Propagation Letters, vol. 15, pp. 1196-1199, 2016.

[23] M. S. Khan, A. Capobianco, S. M. Asif, D. E. Anagnostou, R. M. Shubair and B. D. Braaten, "A Compact CSRREnabled UWB Diversity Antenna", in IEEE Antennas and Wireless Propagation Letters, vol. 16, pp. 808-812, 2017.

[24] W. T. Li, Y. Q. Hei, H. Subbaraman, X. W. Shi and R. T. Chen, "Novel Printed Filtenna With Dual Notches and Good Out-of-Band Characteristics for UWB-MIMO Applications", in IEEE Microwave and Wireless Components Letters, vol. 26, no. 10, pp. 765-767, Oct. 2016.

[25] D. A. Ketzaki and T. V. Yioultsis, "Metamaterial-Based Design of Planar Compact MIMO Monopoles", in IEEE Transactions on Antennas and Propagation, vol. 61, no. 5, pp. 2758-2766, May 2013.

[26] P. J. Ferrer, J. M. González-Arbesú, and J. Romeu, "Decorrelation of two closely spaced antennas with a metamaterial AMC surface", Microw. Opt. Technol. Lett., vol. 50, pp. 1414-1417, May 2008.

[27] M. M. Bait-Suwailam, M. S. Boybay, and O. M. Ramahi, "Electromagnetic coupling reduction in high-profile monopole antennas using single-negative magnetic metamaterials for MIMO applications", IEEE Trans. Antennas Propag., vol. 58, no. 9, pp. 2894-2902, Sep. 2010.

[28] J. Zhu, B. Feng, L. Deng and S. Li, "Ultrawideband MIMO/diversity antenna using CSRR structure for isolation enhancement", 2015 IEEE 4th Asia-Pacific Conference on Antennas and Propagation (APCAP), Kuta, 2015, pp. 27-29.

[29] N.A. Jan, et al., (2017) "A Compact CSRR Loaded Monopole Antenna with Defected Ground Structure for Mobile WLAN and WiMAX Applications", In: Otung I., Pillai P., Eleftherakis G., Giambene G. (eds) Wireless and Satellite Systems. WiSATS 2016. Lecture Notes of the Institute for Computer Sciences, Social Informatics and Telecommunications Engineering, vol 186. Springer, Cham

[30] S. Papantonis and E. Episkopou,"Compact dual-band printed 2.5-shaped monopole antenna for WLAN applications", Progress In Electromagnetics Research C, Vol. 24, PP. 57-68, 2011.

[31] K. H. Sayidmarie, and L. S. Yahya, "Design and Analysis of Dual-Band Crescent Shape Monopole Antenna for WLAN Applications", International Journal of Electromagnetic and Applications, Vol. 3, No. 4, 20 13, PP 96102.

[32] R. A. Bhatti, J. Choi and S. Park, "Quad-Band MIMO Antenna Array for Portable Wireless Communications Terminals", in IEEE Antennas and Wireless Propagation Letters, vol. 8, pp. 129-132, 2009.

[33] S. Blanch, J. Romeu and I. Corbella, "Exact representation of antenna system diversity performance from input parameter description", in Electronics Letters, vol. 39, no. 9, pp. 705-707, 1 May 2003.

[34] M. R. Sharawi, "Printed MIMO antenna engineering", Norwood: Artech House, 2014.

[35] S. H. Chae, S. Oh and S. Park, "Analysis of Mutual Coupling, Correlations, and TARC in WiBro MIMO Array Antenna", in IEEE Antennas and Wireless Propagation Letters, vol. 6, pp. 122-125, 2007.

[36] C. H. See, R. A. Abd-Alhameed, Z. Z. Abidin, N. J. McEwan and P. S. Excell, "Wideband Printed MIMO/Diversity Monopole Antenna for WiFi/WiMAX Applications", in IEEE Transactions on Antennas and Propagation, vol. 60, no. 4, pp. 2028-2035, April 2012. 